\title{
To the theory of discrete boundary value problems
}

\author{
Oksana A. Tarasova, and Vladimir B. Vasilyev* \\ Chair of Differential Equations, Belgorod State National Research University, ul. Pobedy, 85, Belgorod 308015, Russia
}

Received 7 January 2019, Accepted 5 April 2019

\begin{abstract}
We consider discrete analogues of pseudo-differential operators and related discrete equations and boundary value problems. Existence and uniqueness results for special elliptic discrete boundary value problem and comparison for discrete and continuous solutions are given for certain smooth data in discrete SobolevSlobodetskii spaces.
\end{abstract}

Keywords: Digital pseudo-differential operator, Discrete boundary value problem, Periodic factorization, Discrete solution, Error estimate

AMS subject classification: Primary: 35S15, Secondary: 65T50

\section{Introduction}

As a rule the classical pseudo-differential operator in Euclidean space $\mathbb{R}^{m}$ is defined by the formula [1, 2]:

$$
(A u)(x)=\int_{\mathbb{R}^{m}} \tilde{A}(x, \xi) e^{-i x \cdot \xi} \tilde{u}(\xi) \mathrm{d} \xi,
$$

where the sign $\sim$ over a function denotes its Fourier transform,

$$
\tilde{u}(\xi)=\int_{\mathbb{R}^{m}} u(x) e^{i x \cdot \xi} \mathrm{d} x,
$$

and the function $\tilde{A}(x, \xi)$ is called a symbol of a pseudo-differential operator $A$.

Our main goal here is describing a periodic variant of this definition and studying its certain properties related to solvability of corresponding equations in canonical domains of an Euclidean space. In this paper the main result is related to a comparison of discrete and continuous solutions. We try to preserve maximal correspondence for discrete and continuous cases under digitization, it permits to find more appropriate constructions.

This problem is very large and in our opinion it should include the following aspects according to a lot of physical and technical applications of such operators and related equations:

- finite and infinite discrete Fourier transform as a natural technique for such equations;

- choice of appropriate discrete functional spaces;

- studying solvability for infinite discrete equations;

- studying solvability of approximating finite discrete equations;

- a comparison between continuous and infinite discrete equations;

- a comparison between infinite discrete and finite discrete equations.

This is not completed list of questions for studying which we intend to consider. Some results in this direction were obtained for simplest pseudo-differential operators (Calderon-Zygmund operators [3, 4]) and corresponding equations. Also certain results are related to approximate solutions.

There are few variants of the theory of discrete boundary value problems (see, for example [5,6]), but these theories are related especially to partial differential operators and do not use the harmonic analysis technique. Since the classical theory

*Corresponding author: vladimir.b.vasilyev@gmail.com 
of pseudo-differential operators is based on the Fourier transform we will use the discrete Fourier transform and discrete analogue of pseudo-differential operators which will include discrete analogues of partial differential and some integral convolution operators.

\section{Discrete spaces and digital operators}

\subsection{Discrete Sobolev-Slobodetskii spaces}

Given function $u_{d}$ of a discrete variable $\tilde{x} \in h \mathbb{Z}^{m}, h>0$, we define its discrete Fourier transform by the series:

$$
\left(F_{d} u_{d}\right)(\xi) \equiv \tilde{u}_{d}(\xi)=\sum_{\tilde{x} \in \mathbb{Z}^{m}} e^{i \tilde{x} \cdot \xi} u_{d}(\tilde{x}), \xi \in \hbar \mathbb{T}^{m}
$$

where $\mathbb{T}^{m}=[-\pi, \pi]^{m}, \hbar=h^{-1}$, and partial sums are taken over cubes,

$$
Q_{N}=\left\{\tilde{x} \in h \mathbb{Z}^{m}: \tilde{x}=\left(\tilde{x}_{1}, \cdots, \tilde{x}_{m}\right), \quad \max _{1 \leq k \leq m}\left|\tilde{x}_{k}\right| \leq N\right\} .
$$

We will remind here some definitions of functional spaces [7] and will consider discrete analogue of the Schwartz space $S\left(h \mathbb{Z}^{m}\right)$. Let us denote $\zeta^{2}=h^{-2} \sum_{k=1}^{m}\left(e^{-i h \cdot \xi_{k}}-1\right)^{2}$ and introduce the following.

Definition 1. The space $H^{s}\left(h Z^{m}\right)$ is a closure of the space $S\left(h Z^{m}\right)$ with respect to the norm,

$$
\left\|u_{d}\right\|_{s}=\left(\int_{\hbar \mathbb{T}^{m}}\left(1+\left|\zeta^{2}\right|\right)^{s}\left|\tilde{u}_{d}(\xi)\right|^{2} \mathrm{~d} \xi\right)^{\frac{1}{2}} .
$$

Fourier image of the space $H^{s}\left(h \mathbb{Z}^{m}\right)$ will be denoted by $\tilde{H}^{s}\left(\hbar \mathbb{T}^{m}\right)$.

\subsection{Digital pseudo-differential operators}

One can define some discrete operators for such functions $u_{d}$.

If $\tilde{A}_{d}(\xi)$ is a periodic function in $\mathbb{R}^{m}$ with the basic cube of periods $\hbar \mathbb{T}^{m}$ then we consider it as a symbol. We will introduce a digital pseudo-differential operator in the following way.

Definition 2. A digital pseudo-differential operator $A_{d}$ in a discrete domain $D_{d}$ is called the operator [7],

$$
\left(A_{d} u_{d}\right)(\tilde{x})=\sum_{\tilde{y} \in h \mathbb{Z}^{m}} \int_{\hbar T^{m}} \tilde{A}_{d}(\xi) e^{i(\tilde{x}-\tilde{y}) \cdot \xi} \tilde{u}_{d}(\xi) \mathrm{d} \xi, \tilde{x} \in D_{d} .
$$

We use the class $E_{\alpha}, \alpha \in \mathbb{R},[7]$ with the following condition:

$$
c_{1}\left(1+\left|\zeta^{2}\right|\right)^{\frac{\alpha}{2}} \leq\left|A_{d}(\xi)\right| \leq c_{2}\left(1+\left|\zeta^{2}\right|\right)^{\frac{\alpha}{2}}
$$

and universal positive constants $c_{1}, c_{2}$.

Let $D \subset \mathbb{R}^{m}$ be a domain. We will study the equation:

$$
\left(A_{d} u_{d}\right)(\tilde{x})=v_{d}(\tilde{x}), \quad \tilde{x} \in D_{d},
$$

in the discrete domain $D_{d} \equiv D \cap h \mathbb{Z}^{m}$ and will seek a solution $u_{d} \in H^{s}\left(D_{d}\right), v_{d} \in H_{0}^{s-\alpha}\left(D_{d}\right)[7-9]$.

Earlier some canonical domains [8-16] were considered but in this paper we will discuss the cases $\mathbb{R}^{m}, \mathbb{R}_{+}^{m}$.

\section{Solvability and digital-periodic projectors}

\subsection{Periodic factorization}

This case $\mathbb{R}_{+}^{m}$ is very different from $\mathbb{R}^{m}$, and an ellipticity condition is not sufficient for a solvability. A principal role for the solvability takes a concept of the periodic factorization which is defined for an elliptic symbol.

To describe a solvability picture for equation (3) we introduce the following notations. Let us denote $\Pi_{ \pm}=\left\{\left(\xi^{\prime}, \xi_{m} \pm i \tau\right), \xi_{m} \pm i \tau \in \mathbb{C}, \tau>0\right\}, \xi=\left(\xi^{\prime}, \xi_{m}\right) \in \mathbb{T}^{m}$ 
We will use a special periodic factorization of an elliptic symbol $A_{d}(\xi) \in E_{\alpha}$ :

$$
A_{d}(\xi)=A_{d, \quad+}(\xi) A_{d,} \quad-(\xi)
$$

where the factors $A_{d, \pm}(\xi)$ have some analytical properties in half-strips $\hbar \Pi_{ \pm}$and satisfy certain estimates $[7,8]$.

The special index $æ$ of periodic factorization determines the solvability for equation (3), and for special cases we will describe obtained results $[7,8]$. These cases are distinct. So, if $|æ-s|<1 / 2$ then we have the unique solution:

$$
\begin{gathered}
\tilde{u}_{d}(\xi)=\tilde{A}_{d,+}^{-1}(\xi) P_{\xi^{\prime}}^{\mathrm{per}}\left(\tilde{A}_{d,-}^{-1}(\xi) \widetilde{\ell v_{d}}(\xi)\right), \\
\left(P_{\xi^{\prime}}^{\mathrm{per}} \tilde{u}_{d}\right)(\xi) \equiv \frac{1}{2}\left(\tilde{u}_{d}(\xi)+\frac{h}{2 \pi i} v \cdot p \cdot \int_{-\hbar \pi}^{\hbar \pi} \tilde{u}_{d}\left(\xi^{\prime}, \eta_{m}\right) \cot \frac{h\left(\xi_{m}-\eta_{m}\right)}{2} \mathrm{~d} \eta_{m}\right),
\end{gathered}
$$

for equation (3). But if $æ-s=n+\delta, n \in \mathbb{N},|\delta|<1 / 2$ then there are a lot of solutions,

$$
\tilde{u}_{d}(\xi)=\tilde{A}_{d,+}^{-1}(\xi) X_{n}(\xi) P_{\xi^{\prime}}^{\mathrm{per}}\left(X_{n}^{-1}(\xi) \tilde{A}_{d,-}^{-1}(\xi) \widetilde{\ell v_{d}}(\xi)\right)+\tilde{A}_{d,+}^{-1}(\xi) \sum_{k=0}^{n-1} \tilde{c}_{k}\left(\xi^{\prime}\right) \hat{\zeta}_{m}^{k},
$$

where $X_{n}(\xi)$ is an arbitrary polynomial of order $n$ of variables $\hat{\zeta}_{k}=\hbar\left(e^{-i h \xi_{k}}-1\right), k=1, \ldots, m$, satisfying the condition (2), $\tilde{c}_{k}\left(\xi^{\prime}\right), j=0,1, \ldots, n-1$, are arbitrary functions from $\tilde{H}^{s_{k}}\left(\hbar \rrbracket^{m-1}\right), s_{k}=s-\mathfrak{x}+k-1 / 2$.

\subsection{Approximation schemes}

We will consider the pseudo-differential equation:

$$
(A u)(x)=v(x), \quad x \in D,
$$

and suggest for its solution some computational schemes.

We assume that the symbol $A(\xi)$ of the operator $A$ satisfies the condition:

$$
c_{1}(1+|\xi|)^{\alpha} \leq|A(\xi)| \leq c_{2}(1+|\xi|)^{\alpha},
$$

and it is well-known such a symbol admits factorization,

$$
A(\xi)=A_{+}\left(\xi^{\prime}, \xi_{m}\right) A_{-}\left(\xi^{\prime}, \xi_{m}\right),
$$

with respect to the last variable $\xi_{m}$ with the index æ [1].

Since we know solvability conditions for pseudo-differential equations in $\mathbb{R}^{m}$ and $\mathbb{R}_{+}^{m}[1]$ we will select such discrete pseudo-differential operators which reserve all needed properties of their continuous analogues.

\subsubsection{Equations in a whole space}

Let $P_{h}$ be a restriction operator on $h \mathbb{Z}^{m}$, i.e. for $u \in S\left(\mathbb{R}^{m}\right)$

$$
\left(P_{h} u\right)(x)=\left\{\begin{array}{cc}
u(\tilde{x}), & x=\tilde{x} \in h \mathbb{Z}^{m} ; \\
0, & x \notin h \mathbb{Z}^{m} .
\end{array}\right.
$$

We tried this projector for simplest pseudo-differential operators, namely Calderon-Zygmund operators, these operators can be treated as pseudo-differential operators of order 0 , and we obtained very acceptable results [3, 10-12]. But now we will use another restriction operator.

A construction for the restriction operator $Q_{h}$ for functions $u \in S\left(\mathbb{R}^{m}\right)$ is the following. We take the Fourier transform $\tilde{u}(\xi)$, then its restriction on $\hbar \mathbb{T}^{m}$ and periodically continue it onto a whole $\mathbb{R}^{m}$. Further we apply the inverse discrete Fourier transform $F_{d}^{-1}$ and obtain a discrete function which is denoted by $\left(Q_{h} u\right)(\tilde{x}), \quad \tilde{x} \in h \mathbb{Z}^{m}$. In our opinion the projector $Q_{h}$ is more convenient than $P_{h}$ although the projectors $P_{h}$ and $Q_{h}$ are almost the same according to the following result.

Lemma 1. For $u \in S\left(\mathbb{R}^{m}\right), \forall \beta>0$, we have,

$$
\left|\left(P_{h} u\right)(\tilde{x})-\left(Q_{h} u\right)(\tilde{x})\right| \leq C h^{\beta}, \quad \forall \tilde{x} \in h \mathbb{Z}^{m},
$$

where the constant $C$ depends on $u$ only. 
Further, the symbol $A_{d}(\xi)$ will be defined in the following way. We take a restriction of $A(\xi)$ on the cube $\hbar^{m}$ and periodically extend it onto a whole $\mathbb{R}^{m}$. We consider such $h$-operator as an approximate operator for $A$. So, to find a discrete solution for equation (3) for $D=\mathbb{R}^{m}$ we can use the following discrete equation:

$$
A_{d} u_{d}=Q_{h} v
$$

Its solution is given by the formula,

$$
u_{d}(\tilde{x})=\frac{1}{(2 \pi)^{m}} \int_{\hbar \mathbb{T}^{m}} e^{i \tilde{x} \cdot \xi} A^{-1}(\xi) \tilde{v}(\xi) d \xi, \quad \tilde{x} \in h \mathbb{Z}^{m},
$$

so that we do not need to find an approximate solution for an infinite system of linear algebraic equations like [3, 10]. For our case we need to apply any kind of cubature formulas for calculating the latter integral and a cubature formula for calculating the Fourier transform $\tilde{v}(\xi)$.

According to Lemma 1 one can compare discrete and continuous solutions for enough smooth right-hand sides and symbols.

Theorem 1. If the symbol $A(\xi)$ satisfies the condition and is infinitely differentiable on $\mathbb{R}^{m}$, u is a solution of the equation (4), $u_{d}$ is a solution of the equation (5) then for $v \in S\left(\mathbb{R}^{m}\right)$ we have the following error estimate:

$$
\left|u(\tilde{x})-u_{d}(\tilde{x})\right| \leq C h^{\beta}, \quad \forall \tilde{x} \in h \mathbb{Z}^{m}
$$

for arbitrary $\beta>0$.

Proofs for Lemma 1 and Theorem 1 are given in [17].

\subsubsection{Equations in a half-space}

If we put strong enough restrictions on a right-hand side and factorization elements then one can give a comparison between discrete and continuous solutions.

Lemma 2. If $u \in S\left(\mathbb{R}^{m}\right)$ then the following estimate:

$$
\left|\left(F^{-1} P_{\xi^{\prime}} \tilde{u}\right)(\tilde{x})-\left(F_{d}^{-1} P_{\xi^{\prime}}^{\text {per }} \widetilde{Q_{h} u}\right)(\tilde{x})\right| \leq C h^{\beta}, \quad \tilde{x} \in h \mathbb{Z}_{+}^{m},
$$

holds for $\forall \beta>0$, and the constant $C$ depends on $u$ only.

Starting from Lemma 2 and the Theorem 1 we are able to compare discrete and continuous solutions in a half-space. Below we give this comparison under such conditions when a unique solution exists.

To formulate the following theorem we will describe how we need to choose a right-hand side for solving equation (3). We have the following solution of equation (5):

$$
\tilde{u}(\xi)=A_{+}^{-1}(\xi) P_{\xi^{\prime}} A_{-}^{-1}(\xi) \tilde{\ell v}(\xi),
$$

where $P_{\xi^{\prime}}=\frac{1}{2}\left(I+H_{\xi^{\prime}}\right)$ is a projector defined by the classical Hilbert transform with respect to a variable $\xi_{m}[1]$,

$$
\left(H_{\xi^{\prime}} \tilde{u}\right)(\xi)=\frac{1}{\pi i} v \cdot p \cdot \int_{-\infty}^{+\infty} \frac{\tilde{u}\left(\xi^{\prime}, \eta_{m}\right) d \eta_{m}}{\xi_{m}-\eta_{m}}
$$

$\ell v$ is a continuation of $v$ from $\mathbb{R}_{+}^{m}$ into $\mathbb{R}^{m}$ in corresponding functional space. Since the right-hand side in equation (5) is defined in $h \mathbb{Z}_{+}^{m}$ then we choose $Q_{h}(\ell v)$ instead of $\ell v_{d}$ to obtain the required estimate.

Theorem 2. If the symbol $A(\xi)$ satisfies the condition (6) and is infinitely differentiable in $\mathbb{R}^{m}$ with the factors $A_{ \pm}(\xi)$, $u$ is a solution of the equation (5), $u_{d}$ is a solution of the equation (3) then for $v \in S\left(\mathbb{R}^{m}\right)$ we have the following error estimate:

$$
\left|u(\tilde{x})-u_{d}(\tilde{x})\right| \leq C h^{\beta}, \quad \forall \tilde{x} \in h \mathbb{Z}_{+}^{m}
$$

for arbitrary $\beta>0$.

One can find proofs for Lemma 2 and Theorem 2 in [17]. 


\subsection{Non-trivial case}

We have non-uniqueness of a solution for equation (3) for the case $æ-s=n+\delta, n \in \mathbb{N},|\delta|<1 / 2$. We consider here the case $n=1$.

To obtain the unique solution one needs some additional conditions. Discrete analogues of Dirichlet or Neumann conditions give a very simple case. We will consider here the discrete Dirichlet condition:

$$
\left.u_{d}\right|_{\tilde{x}_{m}=0}=g_{d}\left(\tilde{x^{\prime}}\right)
$$

where $g_{d}$ is a given function of a discrete variable in the discrete hyper-plane $h Z^{\mathrm{m}-1}$.

The condition (8) in Fourier images takes the form:

$$
\int_{-\hbar \pi}^{\hbar \pi} \tilde{u}_{d}\left(\xi^{\prime}, \xi_{m}\right) \mathrm{d} \xi_{m}=\tilde{g}_{d}\left(\xi^{\prime}\right)
$$

and according to the previous theorem we obtain the following integral equation with respect to the unknown $\tilde{c}_{0}\left(\xi^{\prime}\right)$,

$$
\int_{-\hbar \pi}^{\hbar \pi} \tilde{c}_{0}\left(\xi^{\prime}\right) A_{d,+}^{-1}\left(\xi^{\prime}, \xi_{m}\right) \mathrm{d} \xi_{m}=\tilde{f}_{d}\left(\xi^{\prime}\right)
$$

where we have used the following notation,

$$
\tilde{f}_{d}\left(\xi^{\prime}\right)=\tilde{g}_{d}\left(\xi^{\prime}\right)-\int_{-\hbar \pi}^{\hbar \pi} A_{d,+}^{-1}\left(\xi^{\prime}, \xi_{m}\right) X_{1}\left(\xi^{\prime}, \xi_{m}\right) P_{\xi^{\prime}}^{\mathrm{per}}\left(X_{1}^{-1} A_{d,-}^{-1} \widetilde{\ell v_{d}}\right)(\xi) \mathrm{d} \xi_{m},
$$

where $X_{1}(\xi)$ is a polynomial of order 1 of variables $\hat{\zeta}_{k}=\hbar\left(e^{-i h \xi_{k}}-1\right), k=1, \ldots, m$ from the class $E_{1}$.

Let us denote,

$$
\int_{-\hbar \pi}^{\hbar \pi} A_{d,+}^{-1}\left(\xi^{\prime}, \xi_{m}\right) \mathrm{d} \xi_{m} \equiv b_{d}\left(\xi^{\prime}\right)
$$

and assuming that $b_{d}\left(\xi^{\prime}\right) \neq 0$ we will find,

$$
\tilde{c}_{0}\left(\xi^{\prime}\right)=b_{d}^{-1}\left(\xi^{\prime}\right) \tilde{f}_{d}\left(\xi^{\prime}\right) .
$$

Then the solution of the problem (3), (8) is the following,

$$
\tilde{u}_{d}(\xi)=\tilde{u}_{d}(\xi)=\tilde{A}_{d,+}^{-1}(\xi) X_{1}(\xi) P_{\xi^{\prime}}^{\mathrm{per}}\left(X_{1}^{-1}(\xi) \tilde{A}_{d,-}^{-1}(\xi) \widetilde{\ell_{d}}(\xi)\right)+b_{d}^{-1}\left(\xi^{\prime}\right) \tilde{f}_{d}\left(\xi^{\prime}\right) A_{d,+}^{-1}\left(\xi^{\prime}, \xi_{m}\right) .
$$

Thus, we obtain the following result.

Theorem 3. Discrete boundary value problem (3), (8) is uniquely solvable in the space $H^{s}\left(h \mathbb{Z}_{+}^{m}\right)$ for arbitrary right-hand side $v_{d} \in H_{0}^{s-\alpha}\left(h \mathbb{Z}_{+}^{m}\right)$ and arbitrary boundary function $g_{d} \in H^{s-1 / 2}\left(h \mathbb{Z}^{m-1}\right)$.

If the right-hand side is zero, i.e. $v_{d} \equiv 0$, then the formula for the solution is very simplified and looks as follows:

$$
\tilde{u}_{d}(\xi)=b_{d}^{-1}\left(\xi^{\prime}\right) \tilde{g}_{d}\left(\xi^{\prime}\right) A_{d,+}^{-1}\left(\xi^{\prime}, \xi_{m}\right),
$$

and after inverse discrete Fourier transform it will be the following,

$$
u_{d}\left(\tilde{x}^{\prime}, \tilde{x}_{m}\right)=\sum_{\tilde{y}^{\prime} \in h \mathbb{Z}^{m-1}} G_{d}\left(\tilde{x}^{\prime}-\tilde{y}^{\prime}, \tilde{x}_{m}\right) g_{d}\left(\tilde{y}^{\prime}\right) h^{m-1},
$$

where the function $G_{d}(\tilde{x})$ of a discrete variable is defined as inverse discrete Fourier transform of the function,

$$
b_{d}^{-1}\left(\xi^{\prime}\right) A_{d,+}^{-1}\left(\xi^{\prime}, \xi_{m}\right) .
$$

The formula (9) is a discrete analogue of Poisson formula for the Dirichlet problem in a half-space. 


\section{Comparison}

To obtain some comparison between discrete and continuous solutions we will remind how the continuous solution looks. The continuous analogue of the discrete boundary value problem is the following:

$$
\begin{gathered}
(A u)(x)=0, \quad x \in \mathbb{R}_{+}^{m}, \\
u\left(x^{\prime}, 0\right)=g\left(x^{\prime}\right), \quad x^{\prime} \in \mathbb{R}^{m-1} .
\end{gathered}
$$

If the index of factorization equals to $æ$ and $æ-s=1+\delta,|\delta|<1 / 2$ then the unique solution for the problem (10), (11) is constructed by the similar formula:

$$
\tilde{u}(\xi)=b^{-1}\left(\xi^{\prime}\right) \tilde{g}\left(\xi^{\prime}\right) A_{+}^{-1}\left(\xi^{\prime}, \xi_{m}\right)
$$

where,

$$
b\left(\xi^{\prime}\right)=\int_{-\infty}^{+\infty} A_{+}^{-1}\left(\xi^{\prime}, \xi_{m}\right) d \xi_{m}
$$

assuming that $b\left(\xi^{\prime}\right) \neq 0, \forall \xi^{\prime} \in \mathbb{R}^{m-1}$. Let us note that this is simplest variant of Shapiro-Lopatinskii condition [1].

We have the following discrete solution:

$$
\tilde{u}_{d}(\xi)=b_{d}^{-1}\left(\xi^{\prime}\right) \tilde{g}_{d}\left(\xi^{\prime}\right) A_{d,+}^{-1}\left(\xi^{\prime}, \xi_{m}\right),
$$

in which we choose special approximations. We take $g_{d}=Q_{h} g$ and $A_{d, \pm}\left(\xi^{\prime}, \xi_{m}\right)$ we take as restrictions of $A_{ \pm}\left(\xi^{\prime}, \xi_{m}\right)$ on $\hbar \pi^{m}$. Then the periodic symbol,

$$
A_{d}(\xi)=A_{d,+}\left(\xi^{\prime}, \xi_{m}\right) A_{d,-}\left(\xi^{\prime}, \xi_{m}\right)
$$

satisfies all conditions of periodic factorization with the same index æ. Moreover, $\tilde{g}_{d}\left(\xi^{\prime}\right)$ and $A_{d,+}\left(\xi^{\prime}, \xi_{m}\right)$ coincide with $\tilde{g}\left(\xi^{\prime}\right)$ and $A_{+}\left(\xi^{\prime}, \xi_{m}\right)$ respectively on $\hbar \mathbb{\varpi}^{m}$.

Theorem 4. Let $\propto>1$. If $\tilde{g}(\xi)$ is a bounded function then a comparison between solutions of problems (3), (8) and (10), (11) is given in the following way,

$$
\left|\tilde{u}(\xi)-\tilde{u}_{d}(\xi)\right| \leq C h^{-1}, \xi \in \hbar \mathbb{T}^{m}
$$

Proof. For this case we have exact formulas for both continuous and discrete solutions. We will estimate the difference,

$$
\tilde{u}(\xi)-\tilde{u}_{d}(\xi), \xi \in \hbar \mathbb{\varpi}^{m}
$$

we have,

$\tilde{u}(\xi)-\tilde{u}_{d}(\xi)=b^{-1}\left(\xi^{\prime}\right) \tilde{g}\left(\xi^{\prime}\right) A_{+}^{-1}\left(\xi^{\prime}, \xi_{m}\right)-b_{d}^{-1}\left(\xi^{\prime}\right) \tilde{g}_{d}\left(\xi^{\prime}\right) A_{d,+}^{-1}\left(\xi^{\prime}, \xi_{m}\right)=\left(b^{-1}\left(\xi^{\prime}\right)-b_{d}^{-1}\left(\xi^{\prime}\right)\right) \tilde{g}_{d}\left(\xi^{\prime}\right) A_{d,+}^{-1}\left(\xi^{\prime}, \xi_{m}\right), \quad \xi \in \hbar \pi^{m}$,

so that we need to estimate $b^{-1}\left(\xi^{\prime}\right)-b_{d}^{-1}\left(\xi^{\prime}\right)$. Since,

$$
\left|b^{-1}\left(\xi^{\prime}\right)-b_{d}^{-1}\left(\xi^{\prime}\right)\right|=\frac{\left|b\left(\xi^{\prime}\right)-b_{d}\left(\xi^{\prime}\right)\right|}{\left|b\left(\xi^{\prime}\right)\right|\left|b_{d}\left(\xi^{\prime}\right)\right|} \leq C\left|b\left(\xi^{\prime}\right)-b_{d}\left(\xi^{\prime}\right)\right|,
$$

because $\inf \left|b\left(\xi^{\prime}\right)\right| \geq c_{3}$, inf $\left|b_{d}\left(\xi^{\prime}\right)\right| \geq c_{4}$ then we will estimate the latter difference. Simplest considerations lead to the following estimate,

$$
\left|b\left(\xi^{\prime}\right)-b_{d}\left(\xi^{\prime}\right)\right|=\left|\int_{-\infty}^{+\infty} A_{+}^{-1}\left(\xi^{\prime}, \xi_{m}\right) \mathrm{d} \xi_{m}-\int_{-\hbar \pi}^{\hbar \pi} A_{d,+}^{-1}\left(\xi^{\prime}, \xi_{m}\right) \mathrm{d} \xi_{m}\right|=\left|\left(\int_{-\infty}^{-\hbar \pi}+\int_{\hbar \pi}^{+\infty}\right) A_{+}^{-1}\left(\xi^{\prime}, \xi_{m}\right) \mathrm{d} \xi_{m}\right| .
$$


We will estimate one integral only, the second one is almost the same,

$$
\int_{\hbar \pi}^{+\infty}\left|A_{+}^{-1}\left(\xi^{\prime}, \xi_{m}\right)\right| \mathrm{d} \xi_{m} \leq c_{5} \int_{\hbar \pi}^{+\infty}\left(1+\left|\xi^{\prime}\right|+\left|\xi_{m}\right|\right)^{-\circledast} \mathrm{d} \xi_{m}=\frac{c_{5}}{-1}\left(1+\left|\xi^{\prime}\right|+\hbar \pi\right)^{1-\infty} \leq c_{6} h^{-1} .
$$

Therefore if $\tilde{g}\left(\xi^{\prime}\right)$ is a bounded function we have the required estimate.

\section{Conclusion}

This paper is one of first steps for studying discrete boundary value problems and their connections with classical theory of boundary value problems for elliptic pseudo-differential equations. We intend to study more general situations in forthcoming papers and to obtain approximation estimates for comparison of discrete and continuous solutions.

\section{Acknowledgements}

Research supported by the State contract of the Russian Ministry of Education and Science (contract No 1.7311.2017/8.9).

\section{References}

1. Eskin GI (1981), Boundary value problems for elliptic pseudodifferential equations, AMS, Providence.

2. Taylor ME (1980), Pseudo-differential operators, Princeton Univ. Press, Princeton.

3. Vasil'ev AV, Vasil'ev VB (2015), Periodic Riemann problem and discrete convolution equations. Diff Equ 51, 5, 652-660.

4. Vasilyev AV, Vasilyev VB (2015), Discrete singular integrals in a half-space. Current trends in analysis and its applications, Proc. 9th ISAAC Congress, Kraków, Poland, Birkhäuser, Basel, pp. 663-670.

5. Vasilyev AV, Vasilyev VB (2017), Two-scale estimates for special finite discrete operators. Math Model Anal 22, 3, 300-310.

6. Samarskii AA (2001), The theory of difference schemes, CRC Press, Boca Raton.

7. Vasilyev AV, Vasilyev VB (2018), Pseudo-differential operators and equations in a discrete half-space. Math Model Anal 23, 3, $492-506$.

8. Vasilyev AV, Vasilyev VB (2018), On some discrete boundary value problems in canonical domains, in: Differential and Difference Equations and Applications. Springer Proc. Math. Stat, Vol. 230, Springer, Cham, pp. 569-579.

9. Ryaben'kii VS (2002), Method of difference potentials and its applications, Springer-Verlag, Berlin-Heidelberg.

10. Vasilyev VB (2017), Discreteness, periodicity, holomorphy, and factorization, in: C Constanda, M Dalla Riva, PD Lamberti, P Musolino (Eds.), Integral Methods in Science and Engineering, Theoretical Technique, Vol. 1. Birkhauser, Cham, Switzerland, pp. $315-324$.

11. Vasilyev AV, Vasilyev VB (2016), Difference and discrete equations on a half-axis and the Wiener-Hopf method. Azerb J Math 6, $1,79-86$.

12. Vasilyev AV, Vasilyev VB (2016), On solvability of some difference-discrete equations. Opusc Math 36, 4, 525-539.

13. Vasilyev AV, Vasilyev VB (2016), On finite discrete operators and equations. Proc Appl Math Mech 16, 1, 771-772.

14. Vasilyev VB (2017), The periodic Cauchy kernel, the periodic Bochner kernel, and discrete pseudo-differential operators. AIP Conf Proc 1863, 140014-1-140014-4.

15. Vasilyev VB (2018), Discrete pseudo-differential operators and boundary value problems in a half-space and a cone. Lobachevskii J Math 39, 2, 289-296.

16. Vasilyev VB (2018), On discrete pseudo-differential operators and equations. Filomat 32, 3, 975-984.

17. Vasilyev VB (2018), On some approximate calculations for certain pseudo-differential equations. Bull Karaganda Univ Math 3, 91, 9-16.

Cite this article as: Tarasova O.A \& Vasilyev V.B 2019. To the theory of discrete boundary value problems. 4 open, $2,17$. 